\title{
COMPORTAMIENTO HISTÓRICO DE LAS TASAS DE INTERÉS REALES EN MÉXICO, 1951-2001
}

\author{
Fernando Aportela Rodríguez \\ José Antonio Ardavín Ituarte \\ Yyannú Cruz Aguayo*
}

Noviembre, 2001

Documento de Investigación No. 2001-05

Dirección General de Investigación Económica

BANCO DE MÉXICO

* Los autores agradecen los comentarios de Alejandro Werner. Las opiniones contenidas en este documento corresponden exclusivamente a los autores y no representan el punto de vista del Banco de México. 


\title{
COMPORTAMIENTO HISTÓRICO DE LAS TASAS DE INTERÉS REALES EN MÉXICO, 1951-2001
}

\author{
Fernando Aportela Rodríguez \\ José Antonio Ardavín Ituarte \\ Yyannú Cruz Aguayo
}

Noviembre, 2001

Documento de Investigación No. 2001-05

\begin{abstract}
RESUMEN
En este artículo se hace un análisis del comportamiento histórico de las tasas de interés reales de corto y de largo plazo en México en el período comprendido de enero de 1951 a julio de 2001, así como del diferencial de tasas de interés reales de México y de los Estados Unidos durante el mismo lapso. Sin tomar en cuenta el periodo de represión financiera de la década de los setenta, del estudio se desprende la existencia de un piso histórico tanto para las tasas de interés reales como para el diferencial de tasas con los Estados Unidos, mismos que corresponden a la época del desarrollo estabilizador. Hacia finales de 1999 y a mediados de 2001, tanto las tasas de interés reales como el diferencial presentaron niveles similares a dichos pisos. Por otra parte, se analizaron el rendimiento neto o bruto del bono UMS26 y el índice EMBI y la relación de estos dos instrumentos con las tasas de interés reales en la década de los noventa. Asimismo, se llevó a cabo un estudio econométrico de los efectos del riesgo país y de la postura de la política monetaria sobre las tasas de interés internas. Este ejercicio sugiere la existencia de un impacto significativo de dichas variables sobre el nivel de las tasas de interés reales durante el período 1995-2001.
\end{abstract}




\section{COMPORTAMIENTO Histórico DE TASAS DE INTERÉS REALES EN MÉXICO, 1951-2001}

\section{Introducción}

El presente documento contiene una descripción del comportamiento histórico de las tasas de interés reales de corto (28 o 30 días) y largo plazo (364 días) en el periodo que comprende de enero de 1951 a julio de 2001. Las tasas de interés reales se calcularon con la inflación pasada y futura. Para este último cálculo, se utilizó a partir de 1997 la expectativa de inflación de especialistas del sector financiero recabada a través de encuestas levantadas por el Banco de México. Asimismo, se construyeron tasas reales ex-ante, no desestacionalizadas y desestacionalizadas, bajo los dos conceptos de expectativas de inflación. El documento también presenta un análisis histórico del diferencial de tasas de interés entre instrumentos mexicanos y estadounidenses. En todos los casos, el plazo de las tasas de interés es mensual.

Debido a una mayor disponibilidad de información, para la década de los noventa se estudian los rendimientos de un conjunto más amplio de instrumentos de deuda del gobierno mexicano. En primer lugar se examina el comportamiento del rendimiento de los bonos UMS26. Estos son instrumentos que tienen plazo a 30 años, están denominados en dólares, y fueron colocados en el extranjero durante 1996. En segundo lugar, se presenta el índice EMBI. El EMBI es un indicador que refleja las condiciones del riesgo país con base, principalmente, en el rendimiento de los bonos Brady mexicanos. Asimismo, en dicha sección del artículo se estudia el efecto del riesgo país y de la postura de la política monetaria sobre las tasas de interés de 1995 a julio de 2001.

Las estimaciones arrojan que durante el periodo del desarrollo estabilizador las tasas reales de largo plazo se mantuvieron en promedio en 4.21 por ciento. El diferencial promedio entre las tasas reales de largo plazo en México y en los Estados Unidos fue de alrededor de 3.5 por ciento durante dicho lapso. El diferencial promedio durante el año 2000 resultó de 4.1 puntos porcentuales y en junio de 2001, éste había descendido a 3.87 puntos porcentuales.

El resto del documento se estructuró de la siguiente manera: la sección II presenta las definiciones utilizadas en el cálculo de las tasas reales. La sección III describe el comportamiento de las tasas de corto plazo a lo largo del periodo de estudio. La sección IV contiene la evolución de las tasas de largo plazo. La sección V presenta el diferencial entre las tasas de interés reales en México y en los Estados Unidos. La sección VI incorpora el análisis del bono UMS26 y del índice EMBI, así como un estudio econométrico 
del efecto del riesgo país y de la postura de política monetaria sobre el nivel de las tasas de interés reales. Finalmente, la sección VII contiene las conclusiones.

\section{Cálculo de las Tasas Reales de Interés}

A continuación se presentan las definiciones utilizadas en este texto para el cálculo de las tasas reales. Dichas tasas pueden definirse bajo dos tipos de crecimiento de los precios: con la inflación futura (acorde con el supuesto de expectativas racionales) o con la inflación pasada (acorde con el supuesto de expectativas adaptativas). Como se verá más adelante, la divergencia entre los valores de las tasas reales estimadas bajo las dos clases de expectativas se vuelve más marcada durante periodos de inestabilidad macroeconómica.

\subsection{Tasas Reales de Interés Utilizando la Inflación Futura}

Las tasas nominales fueron deflactadas con la inflación observada en el periodo de vencimiento del instrumento (28 o 30 días para las de corto plazo y 364 días para las de largo plazo). Nótese que en caso de que los agentes formaran sus expectativas de inflación de forma racional, un estimador insesgado de dichas expectativas sería la inflación efectivamente observada en el futuro. Por tanto, en este documento se considera a la inflación observada como el indicador de la inflación esperada bajo expectativas racionales. Asimismo, con el propósito de utilizar toda la información relevante, desde mediados de 1997 se utilizaron para deflactar las tasas nominales las expectativas de inflación de los analistas del sector privado. ${ }^{1}$

Las tasas reales de corto plazo se calcularon de acuerdo con dos modalidades: no desestacionalizadas y desestacionalizadas. La diferencia entre estos enfoques reside en la tasa de inflación con la cual se deflactan. En el primer caso se utiliza la inflación mensual esperada $\left(\pi_{t}^{e}\right)$, misma que está sujeta a un comportamiento estacional, ya que el crecimiento de los precios de algunos productos se da con una marcada estacionalidad. En el segundo caso, se usa la inflación mensual esperada promedio $\left(\pi_{t}^{\text {edes }}\right)$. Esta se calcula con base en la inflación anual esperada para los próximos doce meses $\left(\Pi_{t+12}^{e}\right)$ con la siguiente fórmula:

$$
\pi_{t}^{\text {edes }}=\left(1+\Pi_{t+12}^{e}\right)^{\wedge(\# \text { Dias } / 364)}-1
$$

1 Debe destacarse que cuando se utilizan las expectativas de inflación de los especialistas del sector privado, no puede afirmarse con precisión que la formación de dichas expectativas se realice de manera racional o adaptativa. 
El número de días puede ser 28 o 30, según el lapso de vencimiento del instrumento de corto plazo. Para las tasas reales de largo plazo no se hace distinción entre tasas no desestacionalizadas y desestacionalizadas. Ello, toda vez que se utiliza la inflación anual para deflactar el rendimiento, y ésta no presenta un comportamiento estacional.

Así, la tasa de interés real no desestacionalizada se estimó como sigue:

$$
r_{t}=\left[\left(\frac{\left(1+\frac{i_{t}}{(364 / \# \text { Días }}\right)}{\left(1+\pi_{t}^{\mathrm{e}}\right)}\right)^{\wedge(364 / \# \text { Días })}\right]-1
$$

donde $\pi_{t}^{e}$ es la inflación mensual esperada en el mes $t$ para el mes siguiente. Como se mencionó, para las tasas previas a mediados de 1997 se supone que la inflación esperada es igual a la que efectivamente se observó; es decir, $\pi_{t}^{e}=\pi_{t+1}$.

La tasa de interés real desestacionalizada se estima de manera análoga a la del caso anterior, pero sustituyendo en la ecuación (2) la expectativa de inflación para el próximo mes $\boldsymbol{\pi}_{t}^{e}$ por la expectativa de inflación desestacionalizada $\pi_{t}^{\text {edes }}$. Esta última es simplemente la inflación anual esperada en el mes $t$ para los próximos doce meses $\Pi_{t+12}^{e}$, expresada en términos mensuales conforme a la ecuación (1).

Asimismo, la tasa de interés real de largo plazo, bajo la modalidad de inflación futura, se calculó también a partir de la ecuación (2). En este caso ésta se simplifica por tratarse de 364 días. Para su estimación se utiliza la inflación anual esperada en los doce meses siguientes a la emisión del instrumento, $\left(\Pi_{t+12}^{e}\right)$, mediante la siguiente fórmula:

$$
r_{t}=\left[\left(\frac{(1+i)}{\left(1+\Pi_{t+12}^{e}\right)}\right)\right]-1
$$




\subsection{Tasas Reales de Interés Utilizando la Inflación Pasada}

En la modalidad de la inflación pasada, se supone que los agentes utilizan como inflación esperada para el periodo de vencimiento del instrumento el crecimiento de los precios en el periodo anterior a la emisión del mismo. Es decir, las expectativas se forman de manera adaptativa.

Para esta variante, las tasas reales de corto plazo no desestacionalizadas se calcularon con la misma fórmula utilizada para estimar las tasas reales no desestacionalizadas bajo la modalidad de inflación futura (ecuación (2)), con la salvedad de que la inflación utilizada es el crecimiento de los precios durante el mes anterior a la emisión del instrumento, $\pi_{t-1}$. Es decir, si un documento de corto plazo es emitido en enero con plazo de un mes, para calcular el rendimiento real se utiliza el crecimiento en los precios observado durante diciembre. Sustituyendo en la ecuación (2), se obtiene la siguiente expresión:

$$
r_{t}=\left[\left(\frac{\left(1+\frac{i_{t}}{(364 / \# \text { Días }}\right)}{\left(1+\pi_{t-1}\right)}\right)^{\wedge(364 / \# \text { Días })}\right]-1
$$

La desestacionalización de las tasas reales requiere nuevamente la utilización de la ecuación (1), sustituyendo en ésta la inflación anual observada en los doce meses anteriores a la emisión del instrumento. Es decir, se considera la inflación anual reportada en el mes $(t-1): \Pi_{t-1}$. Una vez obtenida la inflación mensual promedio del año anterior, $\pi_{t-1}^{\text {des }}$ se sustituye en la ecuación (4) para obtener la tasa real desestacionalizada.

La tasa de interés real de largo plazo, bajo la modalidad de inflación pasada se calculó con igual procedimiento que en el enfoque de inflación futura. La diferencia estriba en remplazar la ecuación (3) la inflación anual observada durante los doce meses anteriores $\Pi_{t-1}$, en lugar de la expectativa de inflación para los doce meses posteriores a la emisión del documento, $\Pi_{\mathrm{t}+12}^{\mathrm{e}}$. 


\section{Tasas Reales de Interés de Corto Plazo}

El resumen de los principales resultados que arrojó el estudio de las tasas reales de corto plazo se presenta en el Cuadro 1 (para una descripción detallada de las tasas utilizadas, ver el Anexo). Los promedios se estimaron para diferentes periodos. De 1951 a 1969, lapso que puede considerarse del desarrollo estabilizador, el nivel promedio de las tasas de las interés reales fue bajo. En el caso de la modalidad de inflación futura, éste se ubicó entre 0.93 y 1.17 por ciento. Conviene destacar que la tasa nominal de corto plazo representativa durante dicho periodo es la que pagaban las cuentas de ahorro. Sin embargo, los bonos hipotecarios de esa época, si bien considerados de más largo plazo, eran en realidad instrumentos líquidos a la vista. ${ }^{3} \mathrm{El}$ rendimiento nominal promedio de estos bonos fue de alrededor de 8 por ciento de 1951 a 1969. Por tanto, si se utilizara este último instrumento para calcular las tasas reales de corto plazo, los promedios de las mismas aumentarían en cerca de 4 puntos porcentuales.

Cuadro 1. Nivel Promedio de las Tasas de Interés de Corto Plazo (28 y 30 Días)

\begin{tabular}{lccccc}
\hline \hline & Nominales & $\begin{array}{c}\text { Reales, Calculadas con Inflación Futura } \\
\text { No Desestaciona- } \\
\text { lizadas }\end{array}$ & $\begin{array}{c}\text { Desestaciona- } \\
\text { lizadas }\end{array}$ & $\begin{array}{c}\text { No Desestaciona- } \\
\text { lizadas }\end{array}$ & $\begin{array}{c}\text { Desestaciona- } \\
\text { lizadas }\end{array}$ \\
\hline \hline $1951-1969$ & 4.50 & 0.93 & 1.17 & 0.85 & 0.39 \\
$1970-1979$ & 6.40 & -7.97 & -8.21 & -7.65 & -6.51 \\
$1980-1987$ & 53.98 & -5.39 & -2.48 & -4.42 & 3.71 \\
$1988-1989$ & 57.34 & 35.93 & 49.38 & 28.27 & 11.87 \\
$1990-1994$ & 19.74 & 5.45 & 4.31 & 5.18 & 5.14 \\
1995 & 48.44 & 4.52 & 20.50 & 3.56 & 22.05 \\
$1996-1998$ & 25.21 & 6.31 & 10.41 & 5.27 & 4.23 \\
1999 & 21.34 & 7.18 & 8.98 & 9.21 & 6.18 \\
2000 & 15.25 & 5.60 & 6.73 & 6.20 & 6.40 \\
$2001 *$ & 13.62 & 6.70 & 6.78 & 10.82 & 7.16 \\
\hline \hline * Hasta julio & & & &
\end{tabular}

El periodo que comprende de 1970 a más de la mitad de la década de los ochenta se caracterizó por mercados financieros reprimidos y alta inflación. Esto se debió en gran parte a la necesidad de financiar déficit públicos elevados mediante mecanismos como el encaje legal. Así, durante la década de los

2 Como se mencionó, la periodicidad de las tasas de interés es mensual para todos los casos.

3 Ver Romeu C. Antonia (1985). 
setenta, las tasas reales de corto plazo fueron negativas. Para el periodo 1970-1979 las tasas reales de corto plazo se ubicaron en niveles entre -6.51 y -8.21 por ciento.

El panorama cambió durante los años ochenta. Si bien todavía hasta 1988 se observó la presencia de represión financiera, las tasas de interés nominales tuvieron un aumento sustancial. El promedio pasó de 6.40 por ciento en la década de los setenta a más de 50 por ciento de 1980 a 1989 (esto se debió a las elevadas tasas de inflación que se registraron). Asimismo, destaca el hecho de que a partir de agosto de 1982 se introdujeron los Certificados de la Tesorería de la Federación (Cetes) los cuales, si bien con algunas limitaciones, reflejaban más adecuadamente las condiciones monetarias en la economía.

Durante el periodo 1980-1987, las tasas de interés reales de corto plazo fueron negativas, excepto en el caso de las tasas desestacionalizadas con la modalidad de inflación pasada (el promedio de esta última fue 3.71 por ciento). Los rendimientos negativos se debieron, junto con la represión financiera, al comportamiento creciente de los precios durante el periodo y, especialmente, a la aceleración de la inflación a partir de 1986.

Los dos últimos años de la década de los ochenta se consideran por separado debido a las medidas aplicadas para lograr la estabilización de la economía, en especial la introducción del Pacto de Solidaridad Económica el 15 de diciembre de 1987. En esas circunstancias, las tasas de interés reales aumentaron sustancialmente. De 1988 a 1989, bajo la modalidad de inflación futura, la tasa real promedio de corto plazo se ubicó entre 35.93 y 49.38 por ciento. Con la modalidad de inflación pasada, en ese lapso las tasas reales fueron menores, ubicándose entre 11.87 y 28.27 por ciento. La amplia diferencia en el nivel de las tasas reales para las dos modalidades puede ser atribuible al rápido descenso de la inflación en los años posteriores al Pacto y, por ende, a errores de predicción de la misma.

Durante los años noventa, las tasas de interés reales de corto plazo presentaron un comportamiento diferenciado. De 1990 a 1994 experimentaron una reducción importante y el promedio de éstas se ubicó entre 4.31 y 5.45 por ciento. Asimismo, cabe destacar que las diferencias entre los cuatro tipos de tasas reales fueron pequeñas durante dicho periodo.

En 1995, las tasas de interés reales de corto plazo promedio no desestacionalizadas no mostraron un aumento y, de hecho, experimentaron reducción. Sin embargo, ese promedio oculta la importante variación observada durante dicho año. Durante los primeros meses de 1995, las tasas de interés reales de corto plazo fueron negativas, mientras que en los meses posteriores las tasas resultaron elevadas. Si se excluye para el cálculo del promedio de las tasas reales a los primeros tres meses y a los primeros cuatro 
meses del año en el caso de la modalidad de inflación futura y pasada, respectivamente, las tasas reales no desestacionalizadas se ubicaron entre 11.63 y 13.76 por ciento. Asimismo, en los primeros meses de 1995, las tasas reales desestacionalizadas alcanzaron niveles superiores en promedio a 50 y 60 por ciento bajo la modalidad de inflación futura y de inflación pasada, respectivamente, y las tasas se mantuvieron elevadas durante el resto del año.

Las tasas de interés reales promedio en el periodo 1996-1998 se caracterizaron por niveles de entre 5.27 y 6.31 por ciento en el caso no desestacionalizado. En cuanto al cálculo desestacionalizado, el promedio de éstas se ubicó entre 4.23 y 10.41 por ciento. Asimismo, cabe destacar que después de la crisis rusa de agosto de 1998, las tasas de interés reales aumentaron a niveles similares a los registrados durante los últimos tres trimestres de 1995. Así, de agosto a diciembre de 1998, el promedio de las tasas reales no desestacionalizadas fue de 11.41 a 14.12 por ciento. Por otra parte, los rendimientos desestacionalizados se ubicaron entre 17.79 y 19.04 por ciento en el mismo periodo.

Durante 1999, las tasas reales de corto plazo fueron en promedio superiores a las anteriores a la crisis rusa. Sin embargo, éstas mostraron un descenso significativo y al cierre del año se ubicaron en niveles inferiores a los alcanzados en julio de 1998. De hecho, en diciembre de 1999 las tasas reales de corto plazo fueron menores que 6 por ciento en el caso de las expectativas racionales e inferiores a 5 por ciento bajo expectativas adaptativas. Durante 2000 se observaron niveles promedio más bajos que los de 1999. Las tasas reales a 28 días se ubicaron en promedio entre 5.6 y 6.7 en términos no desestacionalizados y entre 6.2 y 6.4 en términos desestacionalizados para expectativas racionales y adaptativas, respectivamente. Sin embargo, las tasas reales mostraron un aumento significativo a partir de octubre de 2000, cerrando el año en niveles superiores a 10 por ciento. Posteriormente a febrero de 2001 las tasas reales experimentaron una caída significativa, con lo cual, para julio, las tasas reales desestacionalizadas se ubicaron respectivamente en 2.8 y 3.5 por ciento, bajo la modalidad de inflación futura e inflación pasada.

A continuación se presentan las Gráficas 1 y 2 que incluyen el comportamiento de las tasas de interés reales bajo las dos modalidades descritas. 


\section{Gráfica 1. Tasa de Interés Real de Corto Plazo (28 o 30 Días)}

\section{Modalidad de Inflación Futura}
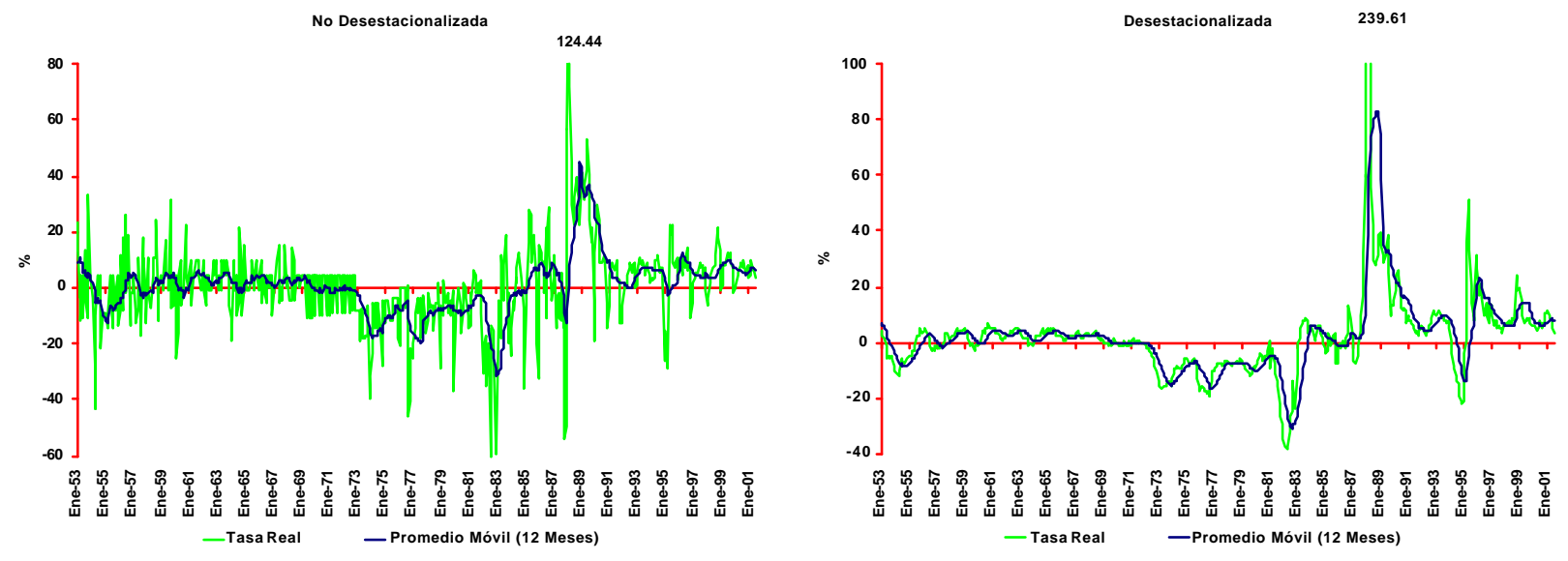

Gráfica 2. Tasa de Interés Real de Corto Plazo (28 o 30 Días)

Modalidad de Inflación Pasada
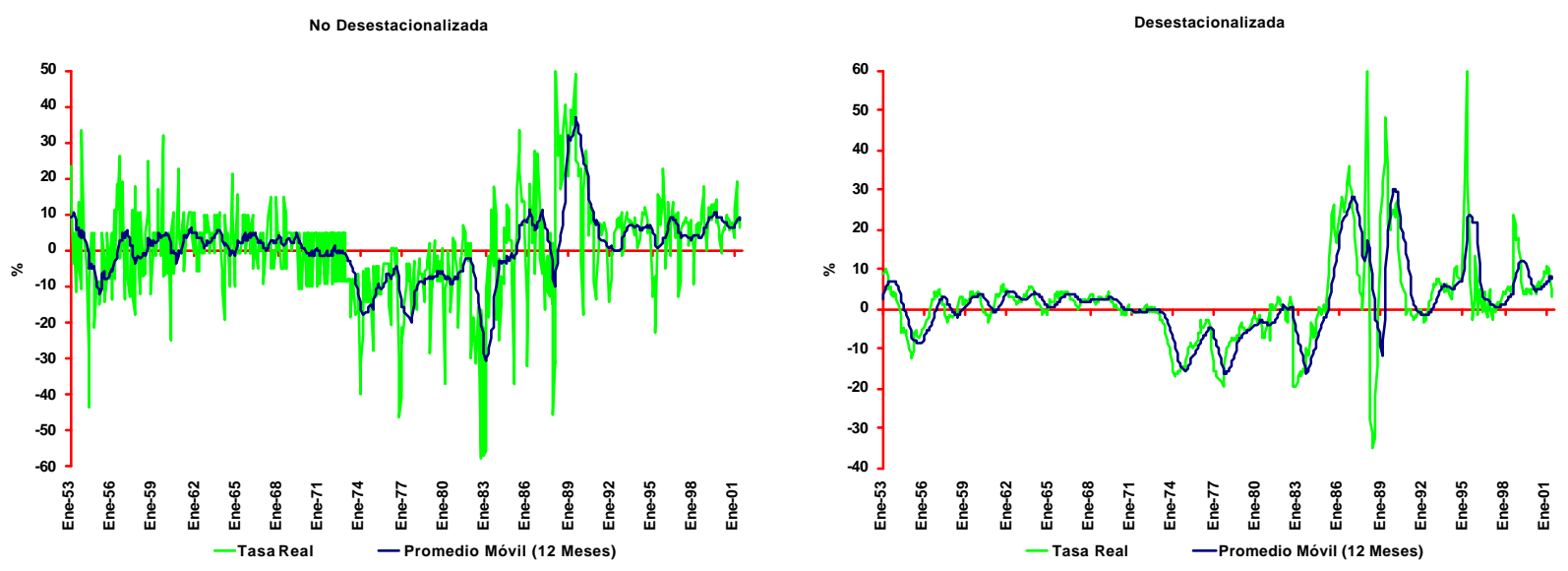

Como se aprecia en las gráficas anteriores, el máximo histórico de las tasas reales de corto plazo bajo la modalidad de inflación futura se observó en febrero de 1988, mes en el que las tasas no desestacionalizadas alcanzaron un nivel de 124.4 por ciento y 239.6 por ciento las desestacionalizadas. Por otra parte, las tasas reales no desestacionalizadas bajo la modalidad de inflación pasada registraron un máximo de 52.3 por ciento, el cual también se observó en febrero de 1988. El nivel máximo de la tasa de corto plazo desestacionalizada bajo esta modalidad fue de 61.68 por ciento, alcanzado en marzo de 1995. 


\section{Tasas de Interés Reales de Largo Plazo}

El nivel promedio de las tasas de interés reales de largo plazo, al igual que el de las de corto plazo, fue positivo en el periodo 1951-1969 (para una descripción detallada de las tasas utilizadas, referirse al Anexo). Éste se ubicó entre 4.29 y 5.11 por ciento bajo las dos modalidades (Cuadro 2). Es conveniente reiterar que hasta mediados de 1963 el instrumento más representativo de largo plazo fue el bono hipotecario, mismo que era líquido en la práctica. ${ }^{4}$

Cuadro 2. Promedio de las Tasas de Interés de Largo Plazo (265 o 364 Días)

\begin{tabular}{lccc}
\hline \hline & Nominales & \multicolumn{2}{c}{ Reales, Modalidad de Inflación: } \\
\cline { 3 - 4 } & & Futura & Pasada \\
\hline \hline & & & \\
$1951-1969$ & 8.70 & 5.11 & 4.29 \\
$1970-1979$ & 13.63 & -2.34 & -0.57 \\
$1980-1987$ & 41.01 & -19.44 & -13.83 \\
$1988-1989$ & 31.77 & 6.93 & -15.55 \\
$1990-1994$ & 17.16 & 0.09 & 0.93 \\
1995 & 43.35 & 6.40 & 7.30 \\
$1996-1998$ & 27.50 & 9.25 & 3.08 \\
1999 & 24.01 & 9.12 & 6.34 \\
2000 & 16.94 & 7.13 & 6.79 \\
$2001 *$ & 15.28 & 7.36 & 7.74 \\
\hline \hline *Hasta julio. & & &
\end{tabular}

De 1970 a 1979, el promedio de las tasas de interés reales de largo plazo fluctuó entre -0.57 y -2.34 por ciento. Ello como reflejo de la represión financiera y de la elevada inflación durante el periodo. El comportamiento no fue muy distinto entre 1980 y 1987. En estos últimos años, las tasas de interés reales, bajo las dos modalidades, resultaron negativas. Debe destacarse que no sólo las tasas reales siguieron siendo negativas, sino que éstas aumentaron en valor absoluto. De 1980 a 1987, el promedio de las tasas reales de largo plazo se ubicó entre -19.44 y -13.83 por ciento.

Durante 1988 y 1989, las tasas de interés reales de largo plazo mostraron un comportamiento diferenciado para las dos modalidades utilizadas. En el caso de inflación futura, la tasa de interés real promedio para el periodo fue de 6.93 por ciento. En contraste, bajo inflación pasada la tasa real fue -15.55 por ciento. Esto se debió a que la inflación se redujo de forma importante en un lapso breve.

4 De agosto de 1989 a diciembre de 1994 las tasas de largo plazo fueron las de los Certificados de Depósito de 180 a 275 días. Asimismo, se suspendió la emisión de Cetes a un año durante abril, mayo, junio, noviembre y diciembre de 1995 y en los últimos tres meses de 1998. 
El promedio de las tasas de interés reales para el periodo de 1990 a 1994 oculta variaciones anuales importantes. En el caso de la modalidad de inflación futura, la tasa real de largo plazo fue positiva de 1990 a 1993, alcanzando un nivel máximo de 7.49 por ciento en 1993. Sin embargo, ésta resultó negativa en 1994, ubicándose en -15.55 por ciento. Lo anterior refleja lo inesperado de la magnitud de la crisis de 1995, así como la severidad del repunte inflacionario en dicho año. Con el enfoque de inflación pasada, el promedio de la tasa de interés real de largo plazo fue negativo tanto en 1991 como en 1992. En 1993 y 1994, esta tasa registró un promedio cercano a 5 por ciento.

Mientras que durante 1995 la tasa de interés real promedio de corto plazo aumentó a niveles superiores a 20 por ciento, la de largo plazo se ubicó entre 6.4 y 7.3 por ciento en promedio. Lo señalado proporciona evidencia de que la volatilidad de las tasas de largo plazo es inferior a la de las de corto plazo.

De 1996 a 1998, el promedio de las tasas de interés reales de largo plazo osciló entre 3.08 y 9.25 por ciento. Bajo la modalidad de inflación futura, las tasas de interés reales más altas en ese periodo se observaron en 1996: 11.04 por ciento en promedio. Asimismo, a lo largo de 1998 la tasa de interés de largo plazo flutuó entre 8.64 y 10.47 por ciento en términos reales, bajo las modalidades de inflación pasada y futura, respectivamente.

Durante 1999, las tasas de largo plazo mostraron una reducción casi continua. Sólo en agosto y septiembre de ese año se observaron repuntes importantes en los rendimientos reales. Al término de 1999, las tasas reales de los Cetes a 364 días se ubicaron en niveles cercanos a 6 por ciento. En 2000 las tasas reales de largo plazo promediaron 7.13 y 6.79 por ciento respectivamente bajo las modalidades de inflación futura y pasada. Por su parte, en julio de 2001 se ubicaron en 5.76 para el primer caso y 6.55 por ciento para el segundo.

A continuación se presentan las series históricas de las tasas de interés reales de largo plazo bajo las dos modalidades (Gráfica 3). 


\section{Gráfica 3. Tasa de Interés Real de Largo Plazo (364 Días)}
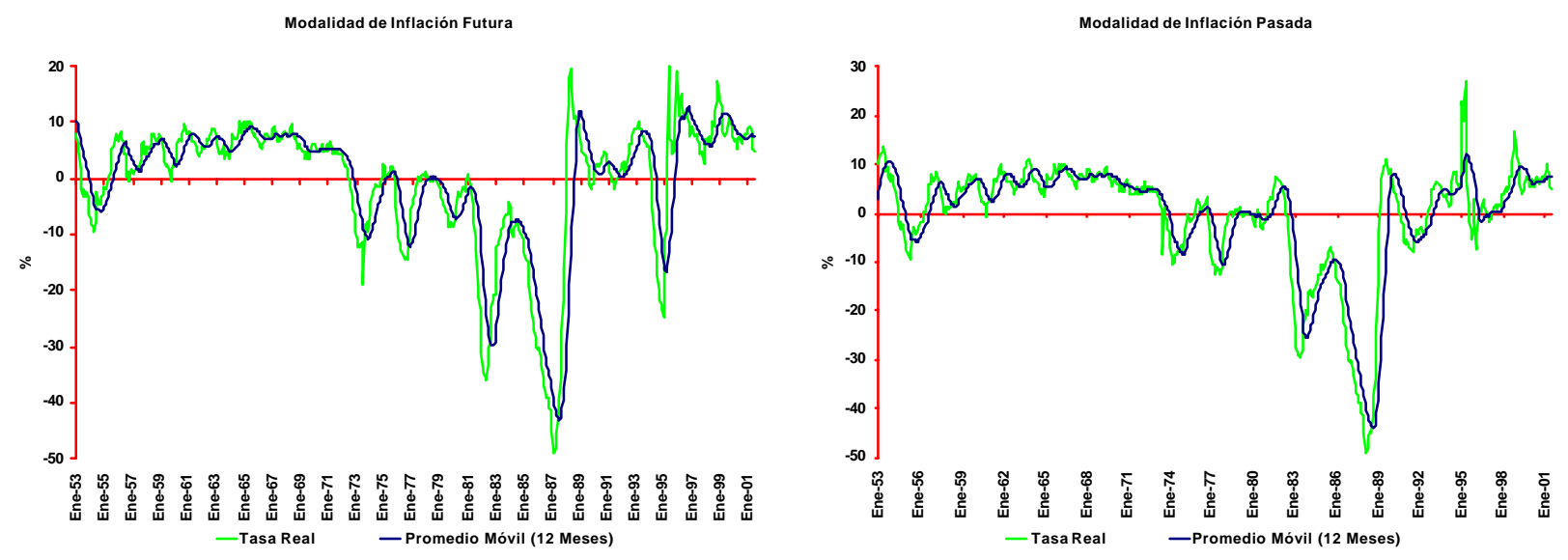

Tanto bajo la modalidad de inflación futura como bajo la de inflación pasada, el nivel mínimo histórico se registró en febrero de 1987 y fue de alrededor de -50 por ciento en los dos casos. Es notorio que los valores de las tasas reales de largo plazo se ubicaron en un intervalo similar bajo las dos modalidades de análisis, situación distinta a la de las tasas de menor plazo presentadas con anterioridad.

\section{Diferenciales de Tasas de Interés México - Estados Unidos}

\subsection{Corto Plazo}

En esta sección se presentan los resultados obtenidos del análisis del diferencial de las tasas de corto plazo en México y en los Estados Unidos. El diferencial se calculó con tasas reales no desestacionalizadas y desestacionalizadas, bajo las dos modalidades de inflación (Cuadro 3). En el periodo que se extiende de 1951 a 1969 el diferencial de tasas osciló entre -0.11 y -0.82 puntos porcentuales. ${ }^{5}$

5 Nuevamente debe mencionarse que la tasa de interés mexicana de corto plazo era la de la cuenta de ahorro. Si se toma en cuenta que los bonos hipotecarios eran en realidad instrumentos líquidos, entonces el diferencial de tasas sería positivo y cercano a 3 puntos porcentuales. 
Cuadro 3. Diferencial Promedio de Tasas de Interés Reales de Corto Plazo (28 y 30 Días) México-Estados Unidos

\begin{tabular}{|c|c|c|c|c|c|}
\hline & \multirow[b]{2}{*}{ Nominales } & \multicolumn{2}{|c|}{ Reales, Calculadas con Inflación Futura } & \multicolumn{2}{|c|}{ Reales, Calculadas con Inflación Pasada } \\
\hline & & $\begin{array}{c}\text { No Desesta- } \\
\text { cionalizada } \\
\end{array}$ & Desestacionalizadas & $\begin{array}{c}\text { No Desesta- } \\
\text { cionalizada }\end{array}$ & Desestacionalizadas \\
\hline $\begin{array}{l}1951-1969 \\
1970-1979\end{array}$ & $\begin{array}{c}1.11 \\
-0.69\end{array}$ & $\begin{array}{l}-0.50 \\
-7.85\end{array}$ & $\begin{array}{l}-0.11 \\
-8.62\end{array}$ & $\begin{array}{l}-0.28 \\
-7.17\end{array}$ & $\begin{array}{l}-0.82 \\
-6.84\end{array}$ \\
\hline 1980-1987 & 43.81 & -11.15 & -8.17 & -9.26 & -1.00 \\
\hline 1988-1989 & 48.99 & 32.07 & 44.57 & 24.61 & 7.77 \\
\hline 1990-1994 & 14.72 & 3.55 & 2.41 & 3.80 & 3.65 \\
\hline 1995 & 42.25 & 0.78 & 17.10 & 0.32 & 18.89 \\
\hline 1996-1998 & 19.75 & 2.56 & 7.30 & 2.09 & 0.94 \\
\hline 1999 & 16.17 & 4.15 & 6.45 & 6.80 & 3.10 \\
\hline 2000 & 8.90 & 2.20 & 3.24 & 3.33 & 3.30 \\
\hline $2001 *$ & 8.92 & 4.30 & 3.60 & 9.73 & 5.66 \\
\hline
\end{tabular}

La represión financiera motivó que el diferencial de las tasas de interés reales en México y en los Estados Unidos fuera negativo de 1970 a 1987. La brecha promedio de las tasas reales de corto plazo de 1980 a 1987 se ubicó entre -11.14 y -1.00 puntos porcentuales.

En los años 1988 y 1989 se observaron los diferenciales promedios de tasas reales más elevados desde 1951. Bajo la modalidad de inflación futura, el diferencial real de corto plazo osciló en esos años entre 32.07 y 45.92 puntos porcentuales. En el caso de la modalidad de inflación pasada, el diferencial promedio de tasas se ubicó entre 7.77 y 24.61 puntos porcentuales.

En el periodo 1990-1994, el diferencial real promedio se movió entre 2.30 y 3.80 puntos porcentuales. Cabe mencionar que estos niveles son comparables (como se apreciará en la sección 5.2) a los registrados por el diferencial de tasas reales de largo plazo en el periodo 1953-1969.

En 1995 los diferenciales de las tasas reales de corto plazo fueron muy elevados. Esto obedeció a la aplicación de una política monetaria restrictiva para propiciar la estabilización de la economía mexicana. Así, el diferencial alcanzó niveles de casi 19 puntos porcentuales en los cálculos desestacionalizados.

El comportamiento del diferencial a lo largo de 1996 y 1997 arroja evidencia de una sustancial mejoría en las condiciones generales de la economía en esos años y de una reducción también significativa en la percepción del riesgo país para México. Entre 1995 y 1997 el diferencial promedio de las tasas reales 
desestacionalizadas se redujo en más de 15 puntos porcentuales bajo la modalidad de inflación futura. Dicha tendencia se vio frenada por el estallido de la crisis rusa en 1998, año en el que el diferencial desestacionalizado aumentó en promedio más de 14 puntos porcentuales, alcanzando niveles superiores a 20 puntos porcentuales.

Durante 1999, los diferenciales de las tasas de interés reales desestacionalizadas de corto plazo mostraron un descenso paulatino, reduciéndose de niveles cercanos a 13 puntos porcentuales a inicios del año a un punto en diciembre. Si bien el diferencial no desestacionalizado es más errático, en el último trimestre del año éste se ubicó en valores inferiores a 10 puntos porcentuales. Durante 2000, el diferencial promedio osciló entre 2.2 y 2.3 puntos porcentuales en la modalidad de inflación futura y resultó de 3.3 puntos utilizando la inflación pasada para el cálculo de las tasas reales. En el transcurso de 2001 se registró un repunte en los diferenciales, por los importantes recortes de las tasas de interés de los Estados Unidos en los meses que antecedieron a la reducción de las mismas en México. Sin embargo, para julio de este año, el diferencial calculado con inflación desestacionalizada, se encontraba en niveles menores que 2.5 puntos porcentuales, tanto para la modalidades de inflación futura como pasada.

A continuación se presentan gráficas con los diferenciales desestacionalizados y no desestacionalizados bajo las dos modalidades de inflación.

\section{Gráfica 4. Diferencial de las Tasas Reales de Corto Plazo (28 o 30 Días) México - Estados Unidos, Modalidad de Inflación Futura}
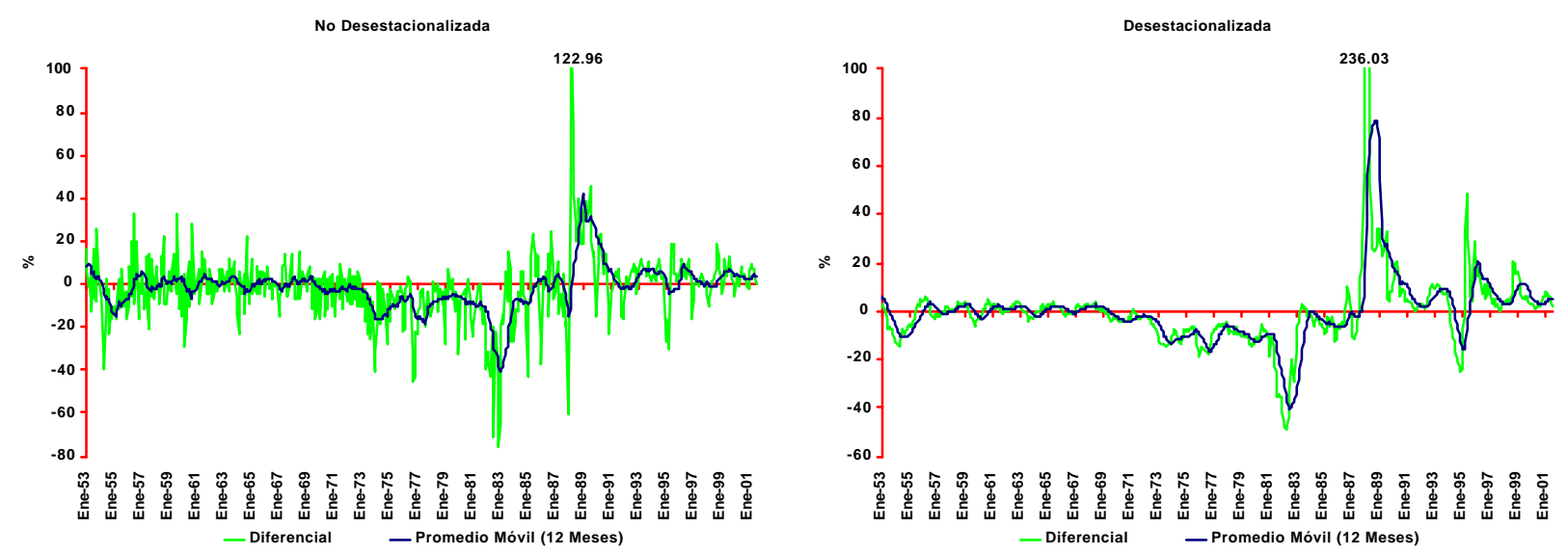


\section{Gráfica 5. Diferencial de las Tasas Reales de Corto Plazo (28 o 30 Días)}

México - Estados Unidos, Modalidad de Inflación Pasada
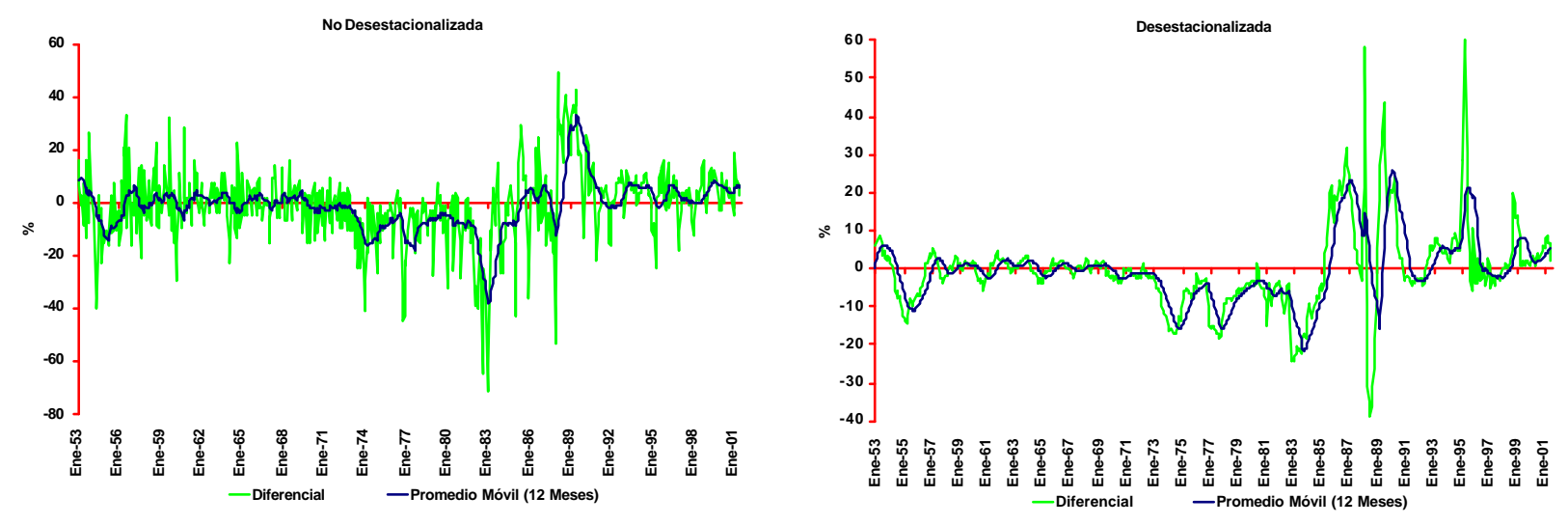

Como se observa en las gráficas anteriores, el diferencial más elevado de tasas reales México - Estados Unidos bajo la modalidad de inflación futura se presentó en febrero de 1988, habiendo sido éste de 122.96 puntos porcentuales para tasas no desestacionalizadas y de 237.72 puntos para tasas desestacionalizadas. En cuanto al criterio de inflación pasada, el diferencial más alto con tasas no desestacionalizadas se observó igualmente en febrero de 1988, con un nivel de 49.06 puntos porcentuales. Con tasas desestacionalizadas, el diferencial alcanzó un máximo de 60.53 puntos porcentuales en marzo de 1995.

\subsection{Largo Plazo}

Los resultados para el diferencial de tasas reales de largo plazo se presentan en el Cuadro 4. En el periodo $1953-1969,{ }^{6}$ el diferencial promedio fue de aproximadamente 5 puntos porcentuales. Sin embargo, a partir de 1970 y hasta 1987, dicho diferencial fue negativo y alcanzó durante la década de los ochenta valores promedio de hasta -24.72 puntos porcentuales.

En 1988 y 1989 el diferencial de largo plazo, bajo la modalidad de inflación futura, fue positivo y se ubicó en 4.09 puntos porcentuales. Sin embargo, al utilizar para el cálculo de las tasas reales la inflación pasada, el diferencial promedio de largo plazo resultó de -19.03 puntos durante ese mismo período. La menor variabilidad de las tasas de largo plazo se vio también reflejada en una volatilidad más reducida del diferencial de rendimientos entre los instrumentos mexicanos y los estadounidenses, en comparación con la del diferencial de tasas de corto plazo.

6 Sólo se contó con observaciones para tasas de largo plazo en los Estados Unidos desde abril de 1953. 
De 1990 a 1994, el diferencial promedio de largo plazo fue negativo y osciló entre -0.64 y -1.98 puntos porcentuales. En 1995, bajo la modalidad de inflación futura, los diferenciales de tasas reales de largo plazo fueron negativos durante la primera parte del año. Sin embargo, a partir de abril, éstos ya fueron positivos y al cierre del año se ubicaron en niveles superiores a 16 puntos porcentuales. En la segunda mitad de 1996 comenzó a observarse una reducción del diferencial de tasas reales de largo plazo, la cual se vio interrumpida en 1998 por la crisis rusa. Como consecuencia de ello, el diferencial pasó de 9.83 a 14.25 puntos porcentuales en un mes. En 1999 el diferencial, bajo la modalidad de inflación pasada, se ubicó en 3.54 puntos, nivel comparable al del promedio de 1953 a 1969. Utilizando la inflación futura el diferencial se ubicó en 7.26 puntos porcentuales. Durante el año 2000, ambos diferenciales resultan comparables con los registrados durante el período del desarrollo estabilizador. Asimismo, si bien el nivel promedio de los diferenciales durante 2001 ha sido superior a los observados entre 1953 y 1969, en julio del presente año fue menor que 4 puntos porcentuales bajo la modalidad de inflación futura y que 6 puntos cuando se utiliza la inflación pasada.

Cuadro 4. Diferencial Promedio de Tasas de Interés Reales de Largo Plazo (364 Días) México - Estados Unidos

\begin{tabular}{lccc}
\hline \hline & Nominales & \multicolumn{2}{c}{ Reales, Modalidad de Inflación: } \\
\cline { 2 - 4 } & & Futura & Pasada \\
\hline \hline $1953-1969$ & 5.11 & 3.44 & 3.48 \\
$1970-1979$ & 6.63 & -2.35 & -0.51 \\
$1980-1987$ & 30.85 & -24.76 & -17.96 \\
$1988-1989$ & 23.68 & 2.73 & -19.03 \\
$1990-1994$ & 11.88 & -1.87 & -0.64 \\
1995 & 37.40 & 0.96 & 4.29 \\
$1996-1998$ & 22.11 & 6.07 & 0.04 \\
1999 & 18.93 & 6.85 & 3.54 \\
2000 & 10.83 & 4.08 & 4.14 \\
$2001 *$ & 11.17 & 4.89 & 6.95 \\
\hline * Hasta julio & & &
\end{tabular}

En la Gráfica 6 se presenta el diferencial de tasas reales de largo plazo bajo las dos modalidades. 


\section{Gráfica 6. Diferencial de las Tasas Reales de Largo Plazo (364 Días)}

\section{México - Estados Unidos}
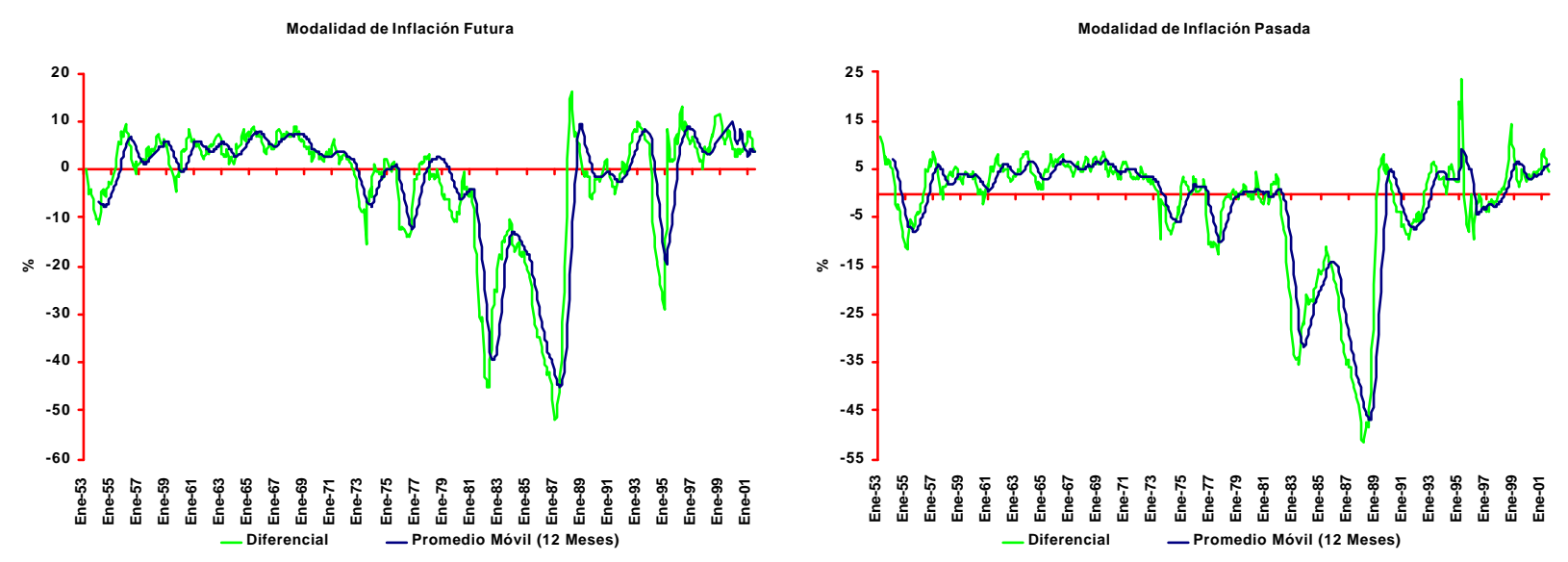

Bajo la modalidad de inflación futura, el mínimo histórico de los diferenciales de tasas de largo plazo México-Estados Unidos se presentó en febrero de 1987 y fue de -50.96 puntos porcentuales. Por otro lado, cuando se utiliza la inflación pasada para el cálculo de las tasas reales, el diferencial resulta de -51.61 puntos en febrero de 1988.

\section{Análisis de los Indicadores del Riesgo País}

En esta sección se presenta el comportamiento de diferentes instrumentos e indicadores que miden el rendimiento de la deuda mexicana denominada en dólares. Complementariamente, estos indicadores también señalan la percepción de los mercados internacionales del riesgo país de México. Los instrumentos aludidos son el UMS26 y el EMBI.

\subsection{Rendimiento del Bono UMS26}

En 1996 el gobierno de México emitió el bono UMS26, ${ }^{7}$ un título denominado en dólares con cupón de 11.5 por ciento y vencimiento a 30 años. El rendimiento de este bono es un indicador de las condiciones de acceso a los mercados financieros internacionales que tiene México, las cuales responden tanto a factores externos como internos.

Inicialmente este instrumento se colocó con un rendimiento promedio de 12.5 por ciento en mayo de 1996. Sin embargo, en diciembre de ese año la tasa de interés pagada por el UMS26 disminuyó 147

7 Por las siglas en inglés deUnited Mexican States y con vencimiento el 15 de Mayo de 2026. 
puntos base. Durante 1997, el rendimiento del aludido bono se ubicó en un intervalo entre 9.43 y 11.04 por ciento (Gráfica 7).

De enero a julio de 1998, la tasa de interés pagada por los bonos UMS26 fue menor que 10 por ciento. Sin embargo, durante agosto ésta experimentó un alza considerable a consecuencia de la crisis rusa. El rendimiento del instrumento pasó de 9.7 por ciento en julio a 11.4 por ciento en agosto y a 12.4 por ciento en septiembre. Eventualmente la tendencia se revirtió, ya que para diciembre de ese mismo año el rendimiento se encontraba en 10.3 por ciento. Durante 1999, la tasa de interés promedio pagada por estos valores fue de 10.22 por ciento y a partir de noviembre de ese mismo año, su rendimiento ha sido menor que 10 por ciento, con excepción de mayo de 2000 cuando promedió 10.1 por ciento. Cabe destacar que las crisis ocurridas desde finales de 2000 y durante 2001 primero en Argentina y después en Turquía, no han afectado la percepción del riesgo país de México, como fue el caso a raíz de la crisis rusa.

En cuanto al rendimiento neto de este instrumento o al diferencial de su tasa con respecto a la del bono a 30 años del Tesoro de los Estados Unidos, éste ha sido generalmente en promedio para todos los años inferior a 5 puntos porcentuales. La diferencia más reducida que se registró en el periodo de análisis fue de 3.5 puntos porcentuales durante 1997, mientras que el diferencial más elevado se observó en septiembre de 1998 cuando alcanzó 7.12 puntos. A partir de noviembre de 1999, dicho diferencial se ha ubicado en niveles cercanos a los 4 puntos porcentuales. Asimismo, en julio de 2001 dicho diferencial fue de 3.4 puntos porcentuales.

Gráfica 7. Rendimiento Bruto y Neto del Bono UMS26
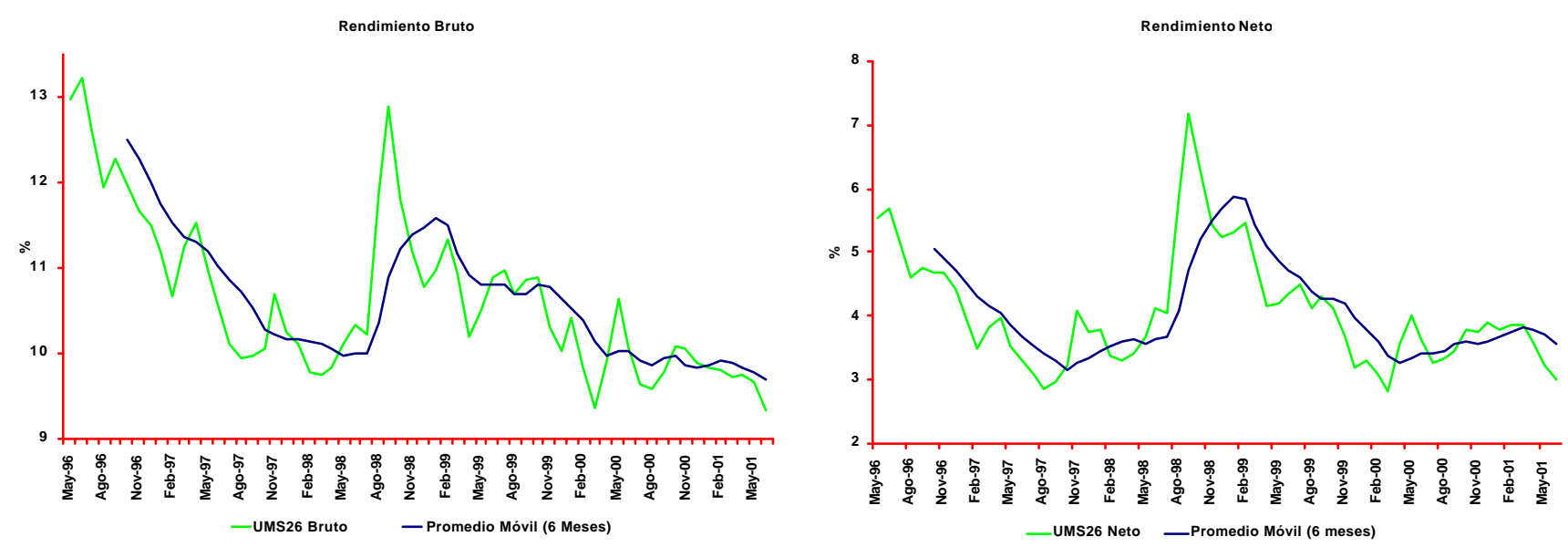


\section{2 $\mathrm{EMBI}^{8}$}

Otro indicador importante del rendimiento de la deuda mexicana denominada en dólares es el índice EMBI, ya señala el costo del financiamiento externo para el país. El EMBI es un índice que mide principalmente el rendimiento total de la deuda externa de los países emergentes en Bonos Brady. Éste indicador fue introducido en 1992 e incluye datos desde 1991 (Gráfica 8).

El EMBI refleja las condiciones del riesgo país determinadas tanto por factores internos como externos. Como puede verse en la Gráfica 8, el comportamiento del EMBI de 1992 a julio de 2001 está influenciado por diferentes acontecimientos que merecen atención. En primer lugar, las reformas económicas de finales de la década de los ochenta y principios de los noventa se tradujeron en fundamentos económicos más sólidos. Ello propició una disminución y estabilidad del valor del EMBI desde los inicios de 1992 hasta finales de 1994. En diciembre de ese año, una elevación drástica del EMBI de 432 puntos base en noviembre a 1,065 puntos base en diciembre, respondió a la profunda crisis económica en que cayó la economía de México. De hecho, el 16 de marzo de 1995 este indicador alcanzó su nivel máximo histórico: 2,426 puntos base. Los valores mínimos de este indicador se registraron en diciembre de 1993 y octubre de 2000 cuando se ubicó en un nivel inferior a los 200 puntos base. A partir de febrero de 2001 el indicador ha vuelto a presentar una reducción importante acercándose a dichos niveles mínimos, al situarse en julio alrededor de los 440 puntos base.

Como se mencionó anteriormente, el EMBI responde también a acontecimientos importantes que ocurren en otros países. Este fue el caso, de las crisis rusa y brasileña. De hecho, la crisis rusa afectó la percepción del riesgo de la mayoría de los países emergentes, entre ellos México. El EMBI del país aumentó de 507 puntos base antes de dicha crisis a 1,175 puntos base durante la misma. La crisis que experimentó Brasil también incidió sobre las economías emergentes, en especial las latinoamericanas. Si bien México fue de los menos afectados por dicha influencia, el indicador del riesgo país aumentó en promedio 83 puntos base durante las cuatro semanas posteriores a dicha crisis.

En suma, las tasas de interés de la deuda externa de México, como lo demuestra el comportamiento del EMBI, responden tanto a las condiciones propias del país. Como a las de otras naciones con características similares. Sin embargo, es importante señalar, como se mencionó para el caso del bono

8 Por sus siglas en inglés: Emerging Markets Bond Index. Este indicador es producido por la empresa JPMorgan, misma que posteriormente introdujo otro indicador denominado EMBI+, que es el más utilizado como medida del rendimiento de la deuda de los mercados emergentes. Este último incluye, en adición a los bonos Brady, préstamos en moneda extranjera y Eurobonos, así como deuda local denominada en dólares. Sin embargo, el presente estudio utiliza el indicador original EMBI por la mayor disponibilidad de datos que permite incorporar al análisis. 
UMS26, que la solidez de la economía mexicana evitó que éstos indicadores se contaminaran a causa de las crisis de Argentina y Turquía de finales de 2000 y principios de 2001. Por otro lado, como se verá en la siguiente sección, las variaciones en el EMBI tienen un efecto significativo sobre los movimientos de la tasa de interés interna.

Gráfica 8. México: EMBI y Promedio Móvil de 30 Días.

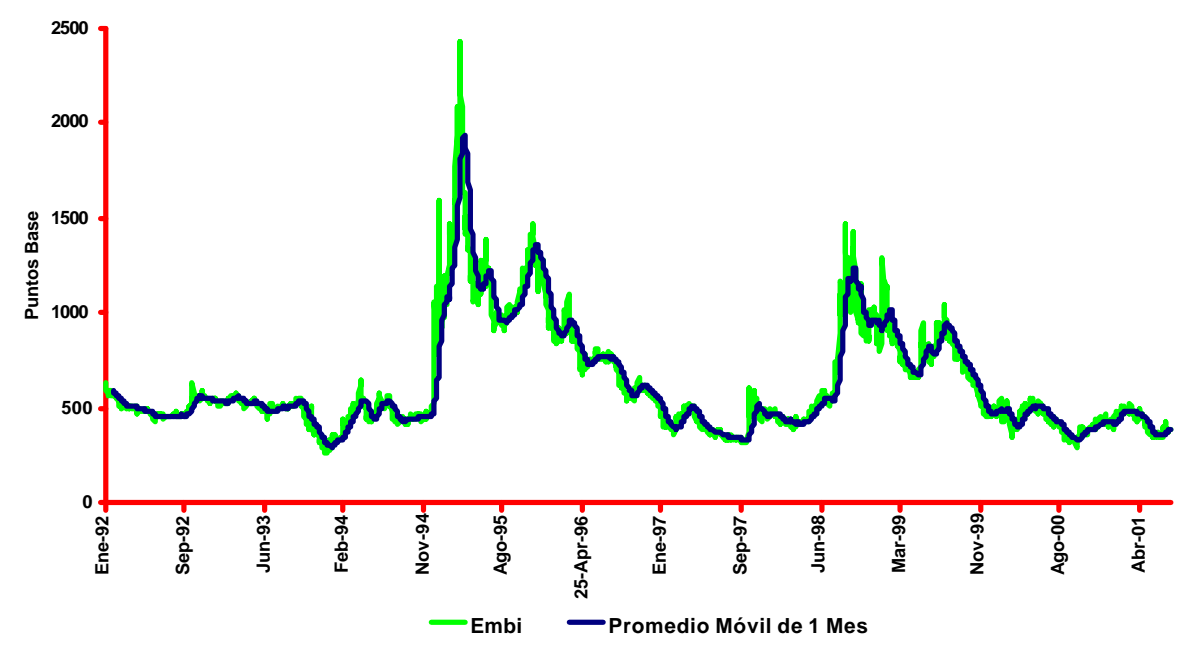

\subsubsection{Efectos del Riesgo País y de la Postura de la Política Monetaria sobre el Nivel de las Tasas de Interés en México}

Las tasas de interés reales pueden experimentar cambios debido a varios factores. Uno de ellos es la postura de la política monetaria del periodo y otro las modificaciones del riesgo país percibido por los extranjeros. ${ }^{9}$ En particular, un método para medir el efecto de dichas modificaciones es estimando la siguiente forma reducida para el cambio de las tasas de interés:

$$
\Delta r_{t}=C_{0}+C_{1} * \Delta E M B I_{t}+C_{2} * \Delta \text { Corto }_{t}+C_{3} * \Delta r_{t-1}+\varepsilon_{t}
$$

donde $\Delta r_{t}$ es el cambio de la tasa de interés real en el periodo t con respecto a su valor en t-1, $\Delta E M B I_{t}$ es la variación del riesgo país en el periodo t con respecto al de t-1, $\Delta$ Corto $_{t}$ es el cambio en el nivel de los saldos acumulados determinado por el Banco de México a través del cuál instrumenta éste su política monetaria. $^{10,11}$

9 Estos factores no pretenden ser una lista exhaustiva de los determinantes de las tasas de interés. En particular, se hicieron ejercicios incluyendo otras variables explicativas, entre ellas las variaciones del tipo de cambio. Los resultados obtenidos no difieren significativamente de los aquí señalados.

10La intervención del Banco de México en el mercado de dinero, conocida como "corto", busca llevar el saldo total observado de las cuentas de la banca comercial al cierre del día hábil anterior al objetivo determinado por el Instituto Central para ese día. Para una descripción detallada del funcionamiento del “corto”, ver Anexo 4 del Informe Anual del Banco de México, 1996. 
La ecuación 5 fue estimada para el periodo que comprende de enero de 1996 a julio de 2001, esto en correspondencia a la fecha del primer "corto" (6 de septiembre de 1995). Asimismo, la inclusión de datos únicamente a partir de 1996, se hizo para eliminar posibles efectos de la crisis del año anterior sobre la estimación de la ecuación. La periodicidad de los datos es diaria. La tasa de interés real utilizada fue la tasa de "fondeo" ${ }^{12}$. Para este ejercicio de simulación se hicieron estimaciones con la tasa real de "fondeo" no desestacionalizada y desestacionalizada bajo las dos modalidades de inflación. ${ }^{13}$ El ejercicio incluyó estimaciones de cuatro tipos de regresiones: la primera incorporó variaciones del riesgo país contemporáneas a las de la variable endógena; en la segunda, el cambio en el EMBI en t-2 se utiliza como variable exógena; en la tercera, el cambio en el EMBI en t-5 se incluye como variable exógena; y en la cuarta, las variables exógenas incluyen la variación en el EMBI en t, t-2 y t-5. Las variables, tanto endógenas como exógenas, se calcularon como cambios absolutos y porcentuales, exceptuando la variable de política monetaria, la cual se introdujo sólo como cambio absoluto.

Los resultados del ejercicio se recogen en los cuadros 5 y 6. Como puede observarse, los cambios en el riesgo país están relacionados positivamente con los del nivel de las tasas de interés reales. De acuerdo con dichos resultados. Cuando se consideran cambios absolutos, un aumento de 100 puntos base en el riesgo país en el periodo t se asocia a un incremento de aproximadamente 78 puntos base en el nivel de la tasa de interés. Asimismo, aumentos sostenidos de 100 puntos base en el riesgo país en t, t-2 y t-5 corresponden a un cambio de aproximadamente 95 puntos base a partir del nivel de la tasa de interés en t.

En la estimación la variable de política monetaria presentó coeficientes con el signo esperado. Es decir, al intensificarse la restricción monetaria las tasas de "fondeo" reales experimentaran un aumento. Asimismo, los coeficientes estimados resultaron significativos estadísticamente en la gran mayoría de los casos. De acuerdo a los resultados explicados, un incremento del "corto" de 10 millones de pesos tendría un efecto alcista de 15 a 25 puntos base sobre el nivel de la tasa de interés real. La variable endógena rezagada no resulta en general significativa.

11 En una especificación alternativa una variable de tendencia fue incluida en las regresiones. Los resultados no se modificaron significativamente.

12 En este caso se utiliza la tasa de "fondeo" bancaria, la cual es representativa de las operaciones de mayoreo realizadas por la banca y casas de bolsa sobre las operaciones en directo y en reporto (de un día hábil bancario) con certificados de depósito, pagarés bancarios y aceptaciones bancarias que hayan sido liquidadas en el sistema de entrega contra pago del INDEVAL (Instituto para el Depósito de Valores, S.A. de C.V.).

13 En este artículo se presentan únicamente los resultados de las tasas reales no desestacionalizadas con el propósito de ahorrar espacio. Los resultados obtenidos con las tasas reales desestacionalizadas no fueron significativamente distintos. 
Cuadro 5. Regresiones de los Cambios Absolutos o Porcentuales de la Tasa Real de “Fondeo" Diaria bajo la Modalidad de Inflación Futura ante Cambios Absolutos o Porcentuales del EMBI

\begin{tabular}{|c|c|c|c|c|c|c|c|c|}
\hline & \multicolumn{4}{|c|}{ Cambio Absoluto } & \multicolumn{4}{|c|}{ "Cambio Porcentual } \\
\hline & (1) & (2) & (3) & (4) & (5) & (6) & (7) & (8) \\
\hline \multirow{2}{*}{$\Delta \mathbf{E M B I}_{\mathrm{t}}$} & 0.7803 & & & 0.6550 & 0.0820 & & & 0.0688 \\
\hline & $(0.0850)$ & & & $(0.0912)$ & $(0.0326)$ & & & $(0.0335)$ \\
\hline$\Delta$ EMBI & & -0.0883 & & 0.0394 & \multirow{2}{*}{\multicolumn{3}{|c|}{$\begin{array}{l}-0.0067 \\
(0.0330)\end{array}$}} & 0.0002 \\
\hline$(\mathrm{t}-2)$ & & $(0.0866)$ & & $(0.0927)$ & & & & $(0.0336)$ \\
\hline$\Delta$ EMBI & & & 0.1679 & 0.2216 & & & 0.0422 & 0.0414 \\
\hline$(\mathrm{t}-5)$ & & & $(0.0879)$ & $(0.0922)$ & & & $(0.0327)$ & $(0.0336)$ \\
\hline $\begin{array}{l}\Delta r \\
(t-1)\end{array}$ & $\begin{array}{l}-0.0332 \\
(0.0271)\end{array}$ & $\begin{array}{c}-0.0108 \\
(0.0279)\end{array}$ & $\begin{array}{c}-0.0132 \\
(0.0280)\end{array}$ & $\begin{array}{c}-0.0538 \\
(0.0290)\end{array}$ & $\begin{array}{c}0.0502 \\
(0.0278)\end{array}$ & $\begin{array}{c}0.0587 \\
(0.0284)\end{array}$ & $\begin{array}{l}0.0604 \\
(.0279)\end{array}$ & $\begin{array}{c}0.0602 \\
(0.0299)\end{array}$ \\
\hline$\Delta$ Corto $_{t}$ & -0.0164 & -0.0234 & -0.0253 & -0.0148 & -0.1730 & -0.2091 & -0.2195 & -0.1726 \\
\hline & $(0.0076)$ & $(0.0075)$ & $(0.0075)$ & $(0.0077)$ & $(0.0795)$ & $(0.0778)$ & $(0.0775)$ & $(0.0863)$ \\
\hline $\mathbf{R}^{2}$ & 0.0680 & 0.0087 & 0.0119 & 0.0525 & 0.0119 & 0.0090 & 0.0113 & 0.0128 \\
\hline DW & 2.0615 & 1.9943 & 1.9657 & 1.9817 & 1.9748 & 1.9686 & 1.9511 & 1.8965 \\
\hline $\mathbf{N}$ & 1268 & 1267 & 1264 & 1132 & 1262 & 1261 & 1258 & 1114 \\
\hline
\end{tabular}

$\overline{\overline{11} \text { Las variables exógenas están también expresadas en cambios absolutos o porcentuales dependiendo del caso. Todas las regresiones incluyen }}$ una constante y una variable de tiempo. Errores estándar en paréntesis. 
Cuadro 6. Regresiones de los Cambios Absolutos o Porcentuales de la Tasa Real

de “Fondeo" Diaria bajo la Modalidad de Inflación Pasada ante Cambios Absolutos o Porcentuales del EMBI

\begin{tabular}{|c|c|c|c|c|c|c|c|c|}
\hline & \multicolumn{4}{|c|}{ Cambio Absoluto } & \multicolumn{4}{|c|}{ Cambio Porcentual } \\
\hline & (1) & (2) & (3) & (4) & (5) & (6) & (7) & (8) \\
\hline \multirow[t]{2}{*}{$\triangle \mathbf{E M B I}_{\mathbf{t}}$} & 0.7501 & & & 0.6307 & 0.2334 & & & 0.1680 \\
\hline & $(0.0826)$ & & & $(0.0892)$ & $(0.4817)$ & & & $(0.3175)$ \\
\hline$\triangle \mathrm{EMBI}$ & & -0.0977 & & 0.0209 & & -0.0772 & & 0.0455 \\
\hline$(\mathrm{t}-2)$ & & $(0.0844)$ & & $(0.0908)$ & & $(0.5051)$ & & $(0.3173)$ \\
\hline$\Delta$ EMBI & & & 0.1046 & 0.1445 & & & -0.0575 & -0.1110 \\
\hline$(\mathrm{t}-5)$ & & & $(0.0853)$ & $(0.0908)$ & & & $(0.3338)$ & $(0.3181)$ \\
\hline $\begin{array}{l}\Delta r \\
(t-1)\end{array}$ & $\begin{array}{l}-0.0266 \\
(0.0271)\end{array}$ & $\begin{array}{l}-0.0041 \\
(0.0280)\end{array}$ & $\begin{array}{l}-0.0040 \\
(0.0280)\end{array}$ & $\begin{array}{l}-0.0432 \\
(0.0291)\end{array}$ & $\begin{array}{l}-0.0129 \\
(0.0269)\end{array}$ & $\begin{array}{l}-0.0031 \\
(0.0295)\end{array}$ & $\begin{array}{l}-0.0005 \\
(0.0267)\end{array}$ & $\begin{array}{c}0.0053 \\
(0.0281)\end{array}$ \\
\hline \multirow[t]{2}{*}{$\Delta$ Corto $_{\mathrm{t}}$} & -0.0162 & -0.0229 & -0.0248 & -0.0146 & -0.6150 & -0.5575 & -0.5998 & -0.7353 \\
\hline & $(0.0074)$ & $(0.0073)$ & $(0.0072)$ & $(0.0076)$ & $(1.1740)$ & (1.1972) & $(0.7913)$ & $(0.8176)$ \\
\hline $\mathbf{R}^{2}$ & 0.0664 & 0.0089 & 0.0102 & 0.0483 & 0.0008 & 0.0002 & 0.0006 & 0.0014 \\
\hline DW & 2.0639 & 1.9970 & 1.9689 & 1.9866 & 1.9821 & 1.8923 & 1.9873 & 1.9310 \\
\hline $\mathbf{N}$ & 1268 & 1267 & 1264 & 1132 & 1262 & 1261 & 1258 & 1114 \\
\hline
\end{tabular}

\section{Conclusiones}

El comportamiento histórico de las tasas de interés reales ha tenido en México etapas bien definidas. En primer lugar, durante el desarrollo estabilizador, los rendimientos reales fueron positivos y de alrededor de 5 por ciento. La época de represión financiera (1970-1987) se caracterizó por rendimientos promedio reales negativos. A continuación, las tasas reales alcanzaron su nivel histórico más elevado durante la aplicación del programa de estabilización de diciembre de 1987.

El comportamiento en la década de los noventa se caracterizó por niveles de las tasas reales moderadamente positivos hasta 1994. En 1995, las tasas aumentaron sustancialmente, en especial las de corto plazo. A partir de 1996 los rendimientos reales descendieron, hasta la crisis rusa de agosto de 1998. Finalmente, si bien las tasas de interés reales fueron elevadas durante 1999, a partir de finales de ese año 
y hasta mediados de 2000 los rendimientos reales mostraron una reducción significativa. En este lapso los niveles se ubicaron por debajo de los prevalecientes antes de la crisis rusa y no fueron muy distintos de los niveles promedio observados de 1951 a 1969. Asimismo, a partir de febrero de 2001 las tasas reales han registrado una baja muy significativa.

Al analizarse el diferencial de las tasas de interés de corto y largo plazo entre México y los Estados Unidos, puede observarse que durante el desarrollo estabilizador los diferenciales promedio fueron aproximadamente de 0.32 y 4.53 puntos porcentuales, respectivamente. Dichos diferenciales se volvieron marcadamente negativos durante la época de la represión financiera en México. Por otro lado, a lo largo de 2000 y hasta abril de 2001, los diferenciales correspondientes fueron en promedio respectivamente del 3.5 y 5.1 puntos porcentuales.

En cuanto los indicadores del rendimiento de la deuda en dólares del gobierno mexicano que dan información sobre la percepción del riesgo país de México, los resultados son como sigue: el diferencial de rendimiento del UMS26 con respecto al bono del Tesoro de los Estados Unidos a 30 años ha sido en promedio inferior a 5 puntos porcentuales. A partir de finales de 1999, éste se ha encontrado en niveles por debajo de 4 puntos, destacando que en este último período dicho rendimiento no fue afectado significativamente por las crisis de otros países emergentes. El índice EMBI y sus variaciones tienen un impacto significativo sobre los cambios de la tasa de interés interna. En el caso del indicador de riesgo, las estimaciones indican que un aumento de 100 puntos base genera un alza de 78 puntos base en la tasa de "fondeo". Asimismo, una política monetaria más restrictiva induce un incremento significativo de las tasas reales.

De la investigación que respalda los resultados aquí comentados destacan algunas observaciones relevantes. En primer lugar, que las tasas reales parecen tener un piso histórico de alrededor de 5 por ciento. Este se observó durante los años del desarrollo estabilizador, periodo que puede tomarse como referencia dada la estabilidad macroeconómica y el crecimiento de la economía prevalecientes durante esa época. Segundo, desde finales de 1999 el nivel del diferencial de tasas reales entre México y los Estados Unidos ha sido similar al del periodo 1951-1969. Durante 2000 no se observó una reducción adicional de las tasas reales de interés, sino más bien un estancamiento y un aumento considerable a partir de octubre de ese año. Sin embargo, a lo largo de 2001 las tasas reales han experimentado una reducción muy importante, ubicándose en julio de dicho año en un nivel cercano a su piso histórico. 


\section{Bibliografía}

[1] Banco de México. SINIEE Sistema Nacional de Información Económica Estructurada. Dirección General de Investigación Económica.

[2] Banco de México. Carpeta de Indicadores Históricos.

[3] Banco de México. Informe Anual 1996.

[4] Federal Reserve Bank of St. Louis. FRED Federal Reserve Economic Data. http://www.stls.frb.org/fred/

[5] Instituto Nacional de Estadística Geografía e Informática. Estadísticas Históricas de México. Tomo II. Agosto 1985

[6] Instituto Nacional de Estadística Geografía e Informática. BIE Banco de Información Económica. http://www.inegi.gob.mx

[7] Romeu C., Antonia. Tasas de Interés Pasivas e Instrumentos de Captación de la Banca Privada y Mixta 19501982. Banco de México, Dirección de Programación Financiera. Octubre 1985.

[8] University of Michigan.Median expected price change next 12 months, Survey of Consumers Reprint from Survey Research Center, University of Michigan at http://www.stls.frb.org/fred/data/business/mich 
Anexo

Instrumentos Utilizados para el Análisis Histórico de las Tasas de Interés

\author{
Cuadro A.1 México
}

\begin{tabular}{|c|c|c|c|}
\hline & & Instrumento & Descripción \\
\hline De & $\mathbf{A}$ & & \\
\hline
\end{tabular}

Tasas de Corto Plazo

\begin{tabular}{|c|c|c|c|c|}
\hline Enero & - & Diciembre & Depósitos de & Instrumento de captación a la vista, que ofreció \\
\hline 1951 & & 1975 & Ahorro & $\begin{array}{l}\text { una tasa constante prácticamente durante todo el } \\
\text { periodo }\end{array}$ \\
\hline Enero & - & Julio & Depósitos a plazo & Saldos sin garantía específica, susceptibles de \\
\hline 1976 & & 1982 & fijo de un mes & $\begin{array}{l}\text { transferirse y darse en garantía, excepto a } \\
\text { instituciones de crédito. }\end{array}$ \\
\hline $\begin{array}{l}\text { Agosto } \\
1982\end{array}$ & - & $\begin{array}{l}\text { Julio } \\
2001\end{array}$ & CETES a 28 días & $\begin{array}{l}\text { Certificados de la Tesorería de la Federación } \\
\text { cuya emisión comenzó en agosto de } 1982 .\end{array}$ \\
\hline
\end{tabular}

Tasas de Largo Plazo (un año)

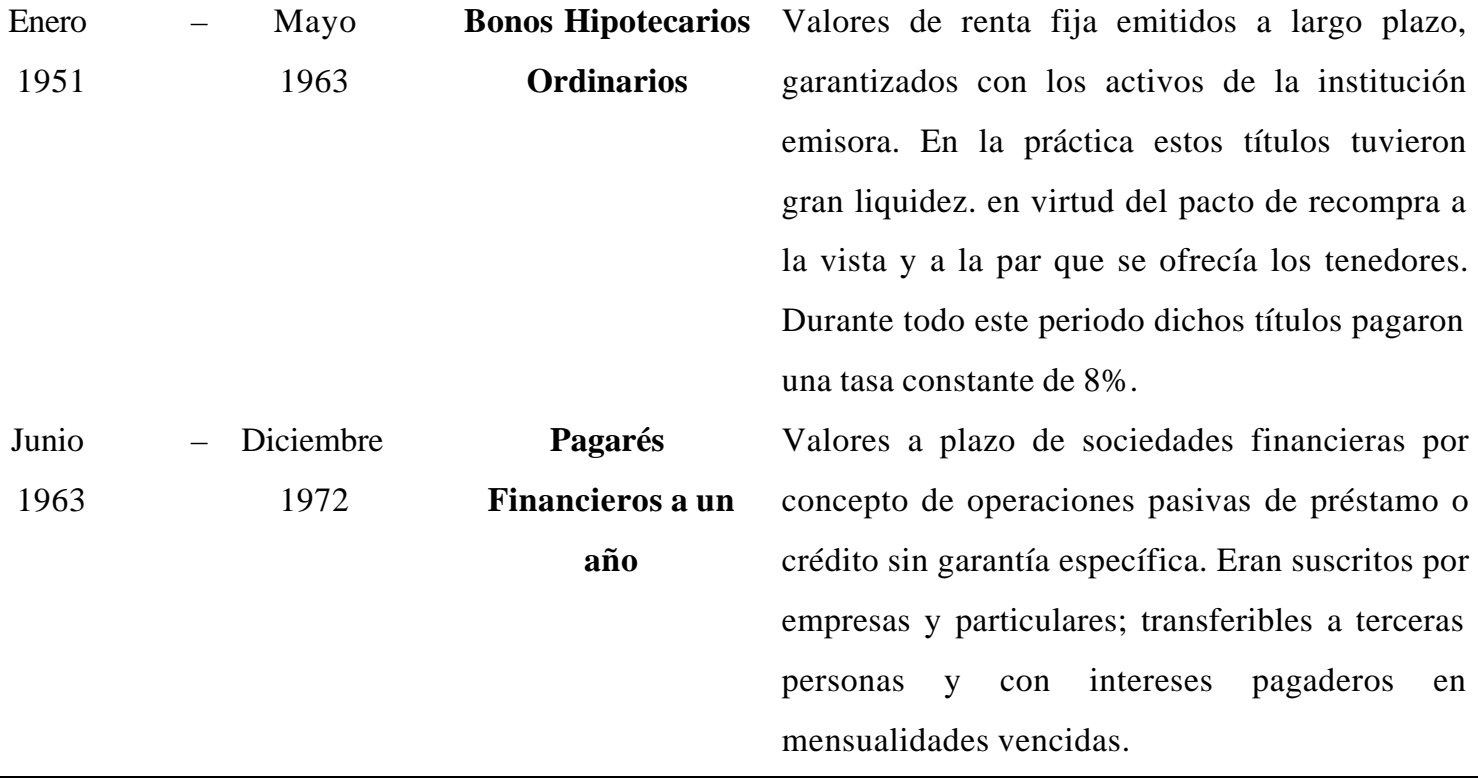




\section{Cuadro A.1, continúa}

\begin{tabular}{|c|c|c|c|c|}
\hline \multicolumn{3}{|c|}{ Período } & \multirow[t]{2}{*}{ Instrumento } & \multirow[t]{2}{*}{ Descripción } \\
\hline De & & $\mathbf{A}$ & & \\
\hline $\begin{array}{r}\text { Enero } \\
1973\end{array}$ & - & $\begin{array}{c}\text { Agosto } \\
1989\end{array}$ & $\begin{array}{c}\text { Certificados de } \\
\text { Depósito a plazo } \\
\text { fijo de } 360 \text { a } 535 \\
\text { días }\end{array}$ & Iguales que los depósitos a plazo fijo a un mes. \\
\hline $\begin{array}{c}\text { Septiembre } \\
1989\end{array}$ & & $\begin{array}{c}\text { Diciembre } \\
1994\end{array}$ & $\begin{array}{c}\text { Certificados de } \\
\text { Depósito a plazo } \\
\text { fijo de } 180 \text { a } 265 \\
\text { días. }\end{array}$ & Iguales que los depósitos a plazo fijo a un mes. \\
\hline $\begin{array}{r}\text { Enero } \\
1995\end{array}$ & - & $\begin{array}{l}\text { Julio } \\
2001\end{array}$ & CETES a 364 días & $\begin{array}{l}\text { Certificados de la Tesorería de la Federación con } \\
\text { vencimiento a un año cuya emisión se inició en } \\
\text { enero de } 1995 .\end{array}$ \\
\hline \multicolumn{3}{|c|}{$\begin{array}{l}\text { Abril, Mayo, Junio, Noviembre y } \\
\text { Diciembre de 1995; } \\
\text { Octubre, Noviembre y Diciembre } \\
\text { de } 1998 .\end{array}$} & $\begin{array}{l}\text { CETES a } 364 \text { días } \\
\text { mercado } \\
\text { secundario }\end{array}$ & $\begin{array}{l}\text { Nota: Para conocer los rendimientos de estos } \\
\text { títulos en los periodos en los que no se } \\
\text { emitieron, se utilizó su rendimiento en el } \\
\text { mercado secundario. Para noviembre y } \\
\text { diciembre de } 1995 \text { se calculó su rendimiento en } \\
\text { el mercado secundario con base en el diferencial } \\
\text { entre éste y el del CETES a } 28 \text { días. }\end{array}$ \\
\hline
\end{tabular}




\section{Cuadro A.2 Estados Unidos}

\begin{tabular}{|c|c|c|}
\hline Período & Instrumento & Descripción \\
\hline De & $\mathbf{A}$ & \\
\hline
\end{tabular}

Tasas de Corto Plazo

Enero 1951 Noviembre de Tasa de Descuento

1965

Diciembre 1965 Abril $2001 \quad$ Certificados de

Depósito a un mes

Tasas de Largo Plazo (un año)

Abril $1953^{14}$ Abril 2001 Treasury Bill de un Bono del Tesoro a un año con tasa de año vencimiento constante

${ }^{14}$ El análisis de largo plazo (un año) comienza con los datos de abril de 1953, por ser ésta la fecha en que se inició la emisión de los Treasury Bills a un año en los Estados Unidos. 